\title{
HRJ
}

v.3 n.14 (2022)

Recebido: 04/11/2021

Aceito: 01/12/2021

\section{Suspensões de Cirurgias em um Hospital Público do Distrito Federal}

\author{
Jaqueline do Vale Bezerra ${ }^{1}$ \\ Raniele Barros de Oliveira ${ }^{2}$ \\ Vanessa da Silva Ferreira ${ }^{3}$ \\ Liliane Travassos Cavalcanti de Souza ${ }^{4}$ \\ Kathiane Magalhães Mendes 5 \\ Taniela Marquez de Paula ${ }^{6}$ \\ Jacqueline Ramos de Andrade Antunes Gomes ${ }^{7}$ \\ Jardel Franco e Silva Anchieta
}

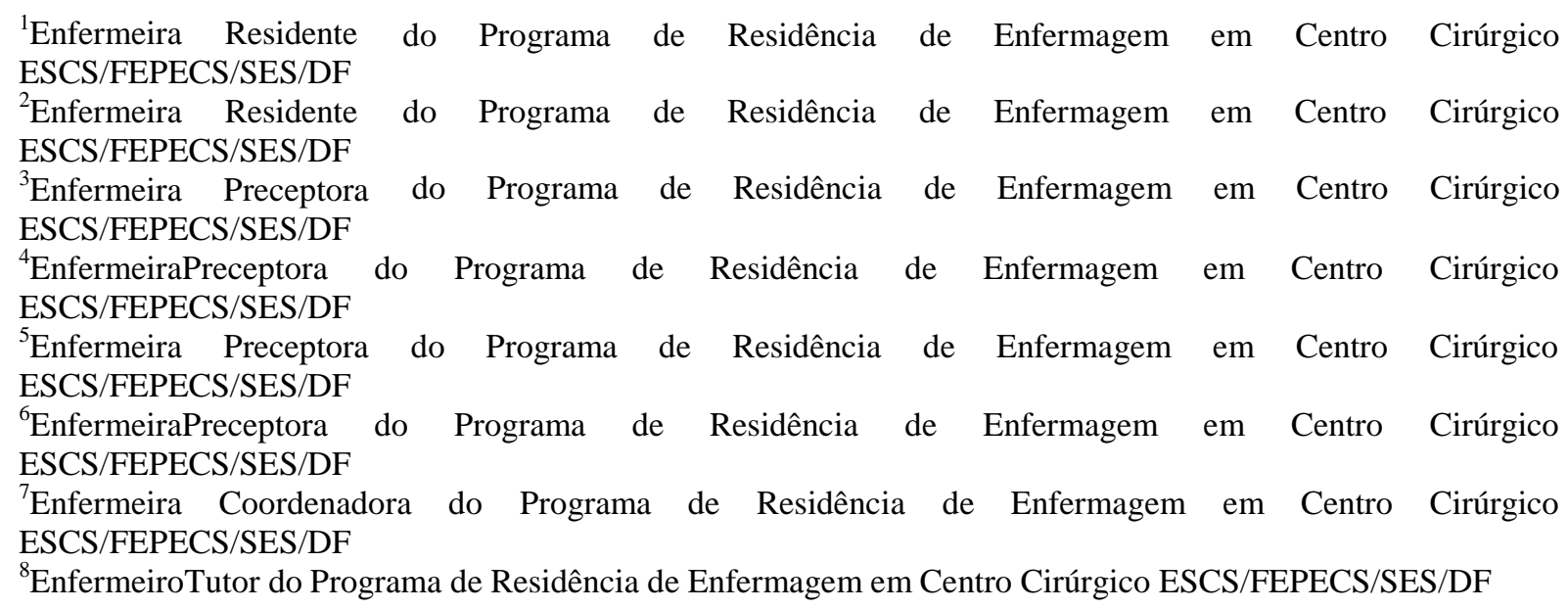

\section{RESUMO}

Objetivos: Mensurar a taxa de suspensão cirúrgica, demonstrar, categorizar, e identificar os cancelamentos cirúrgicos em um hospital público do Distrito Federal. Método: Trata-se de um estudo descritivo, com abordagem quantitativa, e retrospectiva em um centro cirúrgico de um hospital público do Distrito Federal no período de janeiro a dezembro de 2019. Resultado: No período do estudo contabilizou 2.538 cirurgias agendadas de caráter eletivo, dessas, foram realizadas 1.972 e 566 suspensas, totalizando uma taxa anual de suspensão cirúrgica de $22,3 \%$. Os motivos de maior destaque foram relacionados a unidade de saúde 379 (67,0\%), trazendo como principal motivo o avanço de horário, com 128 (22,6\%). Em relação ao paciente das $187(33,0 \%)$ suspensões, as condições clínicas desfavoráveis contabilizaram 82 (14,5\%). Conclusão: O estudo permitiu uma análise situacional do hospital, ressaltando a importância do registro de informações fidedignas e completas para que assim tenha um 
monitoramento dos indicadores de suspensão cirúrgica de forma contínua e com vistas à redução da taxa de suspensão, aumento da qualidade do serviço prestado e consequente minimização dos transtornos causados para os clientes, familiares e instituição.

Palavras-Chaves: Procedimentos Cirúrgicos Eletivos; Indicadores de Qualidade em Assistência à Saúde; Centro Cirúrgico.

\title{
Surgery Suspensions in a Public Hospital in the Federal District
}

\begin{abstract}
Objectives: Measure the rate of surgical suspension, demonstrate, categorize, and identify surgical cancellations in a public hospital in the Federal District. Method: This is a descriptive study, with a quantitative approach, and retrospective in a surgical center of a public hospital in the Federal District from January to December 2019. Result: During the study period, there were 2,538 scheduled surgeries of an elective character, of these, 1,972 and 566 were suspended, totaling an annual surgical suspension rate of $22.3 \%$. The most prominent reasons were related to the health unit 379 (67.0\%), with the main reason being the increase in hours, with $128(22.6 \%)$. Regarding the patient of the 187 (33.0\%) suspensions, the unfavorable clinical conditions accounted for $82(14.5 \%)$. Conclusion: The study allowed a situational analysis of the hospital, emphasizing the importance of recording reliable and complete information so that it can monitor surgical suspension indicators continuously and with a view to reducing the suspension rate, increasing the quality of service provided and consequent minimization of inconvenience caused to clients, family and institution.
\end{abstract}

Keywords: Elective Surgical Procedures; Health Care Quality Indicators; Surgical Center.

\section{INTRODUÇÃO}

O termo cirurgia, também chamado de ato cirúrgico ou procedimento cirúrgico pode ser definido como campo da medicina que visa estudar ou realizar uma intervenção manual ou instrumental no corpo do paciente para diagnosticar, tratar, curar doenças ou traumatismo, de tal modo que melhore a condição clínica geral do individuo ${ }^{1}$.

Segundo as diretrizes de práticas recomendadas da Associação Brasileira de Enfermeiros de Centro Cirúrgico, Recuperação Anestésica, e Centro de Material e Esterilização (SOBECC), as cirurgias programadas com antecedência se classificam como cirurgias eletivas, ou seja, há mais tempo para o paciente e a equipe se prepararem para a intervenção assim reduzindo o risco de intercorrências ${ }^{2}$. 
O ato cirúrgico é um processo que possui várias finalidades, e é considerado indispensável para a manutenção e a recuperação da saúde dos indivíduos. A cirurgia é um acontecimento que demanda de recursos humanos, materiais e tecnológicos, além de promover alterações no perfil psicológico e nos recursos financeiros dos pacientes, familiares e instituição hospitalar. Desta forma, o bem-estar do paciente em situação cirúrgica deve ser o principal objetivo dos profissionais ${ }^{3}$.

O ato cirúrgico vem carregado de emoções e sensações, que associados com o possível cancelamento geram sentimento de frustração, estresse adicional, abalos psicológicos, prejuízo no planejamento da vida diária, traz consigo também problemas para o hospital, gerando atrasos na programação cirúrgica, prejuízos para outros pacientes, ampliação do custo operacional e financeiro, prolongamento do período de internação, uma vez que todos se preparam para um evento que acaba não acontecendo ${ }^{4}$.

As suspensões de cirurgias é um acontecimento comum na programação cirúrgica, podendo ocorrer devido a vários fatores, sejam eles relacionados ao paciente, equipe médica ou de enfermagem, estruturais e institucionais. Os custos desses procedimentos giram em torno de $40 \%$ do total das despesas de um hospital, assim ressaltando a necessidade de ter uma gestão eficaz devido ao fato de ter uma importante função nas unidades prestadoras de serviços de saúde especialmente em instituições públicas onde os recursos são escassos ${ }^{5}$.

No Brasil as taxas de cancelamentos variam de $14,14 \%$ a $27,4 \%{ }^{6}$. Um estudo realizado de janeiro a outubro de 2015 em um hospital da rede pública do Distrito Federal apresentou taxas de suspensões de $33,8 \%{ }^{7}$. As repercussões do cancelamento cirúrgico incidem de forma desfavorável tanto para o paciente, que tem seu vínculo de confiança rompido, como para instituição hospitalar que tem prejuízos quanto ao custo operacional e financeiro ${ }^{8}$.

Portando, devido ao aumento do número de cirurgias eletivas nos últimos anos, a taxa de cancelamento de cirurgias tem sido adotada como um indicador de qualidade da 
assistência. Para tanto, é preciso que os enfermeiros possam estudar esses dados e apresentálos a fim de reduzir as taxas. Desta forma, torna-se necessário e de grande valia a frequência de investigações e levantamento de dados quanto às suspensões cirúrgicas. Contudo, decidiuse elaborar este estudo para verificar quantitativamente as ocorrências desses eventos e, principalmente, as suas causas justificando assim a relevância deste estudo.

Objetiva- se com este estudo mensurar a taxa de suspensão cirúrgica, demonstrar, categorizar, e identificar os cancelamentos cirúrgicos em um hospital público do Distrito Federal.

\section{MÉTODO}

Trata-se de uma pesquisa descritiva, com abordagem quantitativa, e retrospectiva. $\mathrm{O}$ método da pesquisa visa descrever as características de determinada população ou fenômeno ou o estabelecimento de relações entre variáveis 9 .

O estudo foi realizado no centro cirúrgico (CC) de um Hospital Regional do Distrito Federal, onde os dados são registrados em livro pela equipe de enfermagem. O Hospital possui 5 salas de centro cirúrgico, sendo 1 delas destinada a cirurgias de urgências/emergências, e as demais para procedimentos eletivos.

A amostra foi composta por informações disponibilizadas em livro de registro de cirurgias da unidade, onde totalizou 2.538 cirurgias eletivas conforme mapa cirúrgico no período de janeiro a dezembro de 2019. Destas totalizou 566 cancelamentos no período. Como critérios de inclusão foram considerados todas as cirurgias de caráter eletivas que foram canceladas por qualquer motivo e descritas no livro de registros da enfermagem no período de janeiro a dezembro de 2019. Excluíram-se cirurgias de urgências descritas como suspensa, e dados que não estavam claros e/ou incompletos.

O instrumento utilizado para a coleta de dados foi elaborado pelos autores do estudo 
para facilitar a organização, e posteriormente serem lançados em planilhas. Foi utilizado o formulário de forma separada por mês, sendo preenchido com um "x" correspondente a variável que o paciente se encaixava. As variáveis utilizadas como determinantes da pesquisa foram: quanto ao gênero (masculino ou feminino), quanto à faixa etária (crianças $\leq 12$ anos, adolescente 13 a 18 anos, adulto 19 a 59 anos, idoso $\geq 60$ anos), quanto à especialidade (cirurgia geral, ortopedia, ginecologia, mastologia, plástica, proctologia, urologia, vascular e odontologia), quanto às causas relacionadas ao paciente (condição clínica desfavorável, não comparecimento, falta de jejum, recusa a cirurgia, falta de exames, outros), relacionado à unidade (erro na programação cirúrgica, falta de vaga na clínica cirúrgica, falta de vaga na UTI, substituições de paciente, falta de anestesista, avanço de horário, falta de cirurgião, mudança de conduta médica, relacionado à falta de materiais da central de material esterilizado - CME, material da farmácia, não especificado, e outros).

No que diz respeito aos fatores determinantes relacionadas ao paciente, foram consideradas na categoria "outros" aqueles motivos que diferem das demais categorias mencionadas, como por exemplo: internado em outra unidade, óbito de familiar que impossibilitou o comparecimento, administração de medicamentos contraindicado no préoperatório e demais motivos de força maior. Já na categoria “outros" relacionados aos fatores estruturais da unidade de saúde foram considerados motivos que não se encaixavam nos demais, como por exemplo: equipamentos de raio-x e escopia quebrados e/ou manutenção, falta de energia elétrica, e problemas no ar-condicionado.

Os dados foram contabilizados e posteriormente analisados e comparados por meio de frequência simples das variáveis pesquisadas, com auxílio do software IBM SPSS 20.0. As taxas de suspensão cirúrgica foram calculadas dividindo o número de cirurgias suspensas pelo total de cirurgias eletivas, multiplicado por $100^{1,7,6}$.

Esta pesquisa obedeceu aos preceitos éticos e legais, tendo seu projeto submetido à 
apreciação do Comitê de Ética em Pesquisa (CEP) da Fundação de Ensino e Pesquisa (FEPECS), da Secretaria de Estado de Saúde do Distrito Federal, e aprovado sob o número CAAE 32782520.6.0000.8153, conforme preconiza a Resolução no 466/2012, do Conselho Nacional de Saúde, que aborda as diretrizes para pesquisas envolvendo seres humanos ${ }^{10}$.

\section{RESULTADOS}

No período de janeiro a dezembro de 2019, o hospital de estudo apresentou um total de 2.538 cirurgias agendadas de caráter eletivo, dessas, foram realizadas 1.972 e 566 suspensas, totalizando uma taxa anual de suspensão cirúrgica de 22,3\%. A análise dos dados referente aos procedimentos agendados e suspensos foi realizada mensalmente, e as taxas de suspensões mensais estão demonstradas na Figura 1.

No período total do estudo, das 566 cirurgias suspensas, a maioria corresponde ao sexo feminino, totalizando 297 (52,5\%). Quanto à faixa etária dos pacientes, os adultos (19 a 59 anos) foram à maioria totalizando $362(64,0 \%)$.

Figura 1: Taxa de suspensão mensal de janeiro a dezembro, e o total do ano de 2019.

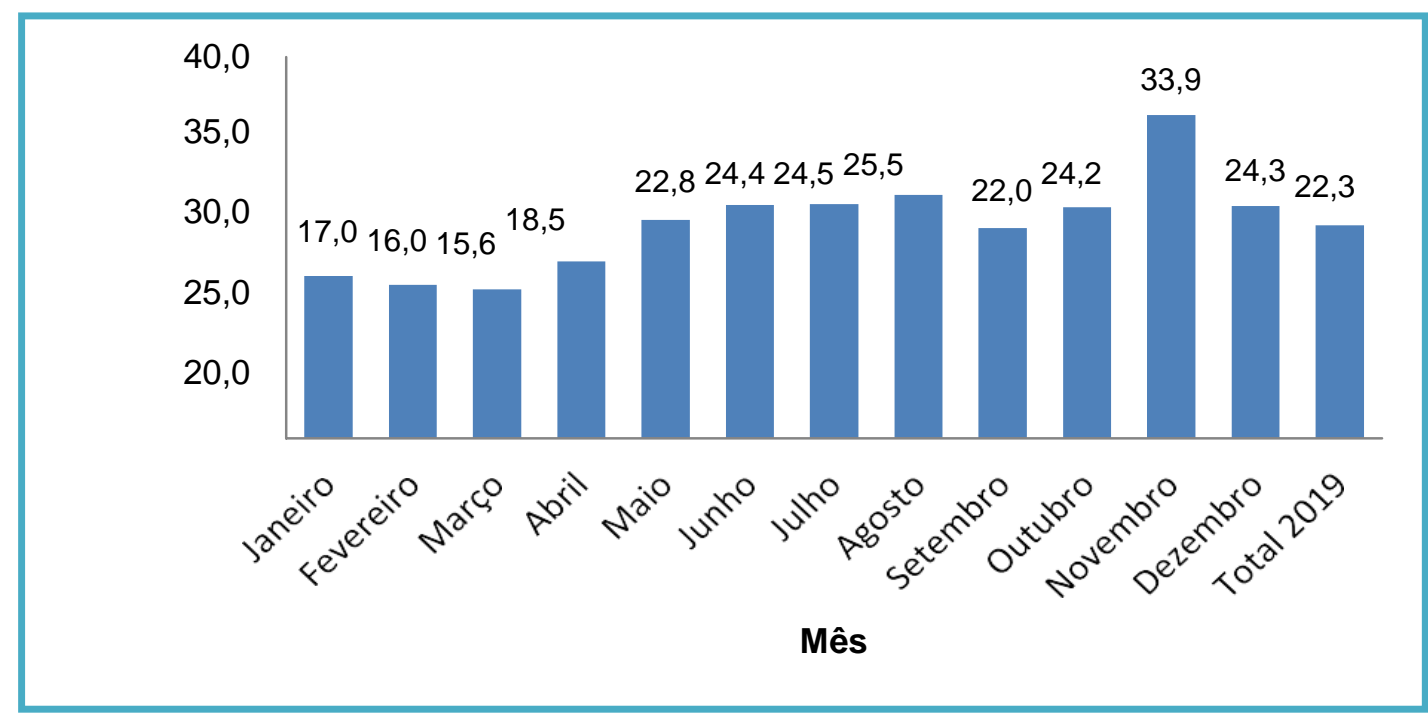

Já em relação à especialidade cirúrgica destaca-se a ortopedia com 671 procedimentos 
agendados, e desses, $243(36,21 \%)$ procedimentos suspensos, seguido pela cirurgia geral com 527 cirurgias agendadas e $117(22,2 \%)$ suspensas. A mastologia apresentou a menor taxa de 137 procedimentos agendados, teve $12(8,8 \%)$ de procedimentos suspensos. O resultado das demais especialidades quanto ao número de cirurgias agendadas, realizadas, suspensas, e suas respectivas taxas podem ser observados na Tabela 1.

Em relação aos meses, observa-se em destaque o mês de novembro com 177 cirurgias agendadas, e 60 procedimentos suspensos, alcançando uma taxa de suspensão de 33,9\%, seguido pelo mês de agosto com 259 procedimentos agendados e $66(25,5 \%)$ suspensos. O mês de menor taxa foi o mês de março com 186 agendamentos e 29 (15,6\%) suspensos. Os demais resultados podem ser observados na Tabela 1.

Tabela 1: Distribuição de cirurgias eletivas programadas, realizadas, suspensas e taxa de suspensão por especialidade de acordo com o número de cirurgias agendadas por cada clínica no ano de 2019.

\begin{tabular}{|c|c|c|c|c|}
\hline Especialidade & (N) Agendadas & (N) Eletivas realizadas & $\begin{array}{c}\text { (N) Cirurgias } \\
\text { Suspensas }\end{array}$ & $(\%)$ \\
\hline Ortopedia & 671 & 428 & 243 & 36,2 \\
\hline $\begin{array}{c}\text { Cirurgia } \\
\text { Geral }\end{array}$ & 527 & 410 & 117 & 22,2 \\
\hline Urologia & 162 & 131 & 31 & 19,1 \\
\hline Odontologia & 33 & 27 & 6 & 18,2 \\
\hline Ginecologia & 451 & 371 & 80 & 17,7 \\
\hline Vascular & 218 & 180 & 38 & 17,4 \\
\hline Proctologia & 64 & 54 & 10 & 15,6 \\
\hline Plástica & 275 & 246 & 29 & 10,5 \\
\hline Mastologia & 137 & 125 & 12 & 8,8 \\
\hline Total & 2538 & $\begin{array}{l}197 \\
2\end{array}$ & 566 & 22,3 \\
\hline
\end{tabular}


Ao analisar as causas de suspensões, das 566 suspensões, 379 (67,0\%) corresponde a causas relacionadas a fatores estruturais, ou seja, relacionados à unidade de saúde, tendo como destaque o motivo "Avanço de horário", cujo totalizou 128 (22,6\%), e o que apresentou menor taxa foi falta de materiais relacionados à farmácia, representando $2(0,4 \%)$.

Já as causas relacionadas a fatores relacionados aos pacientes obtiveram-se 187 (33,0\%), dando destaque à justificativa de "Condição clínica desfavorável” que contabilizou $82(14,5 \%)$ de frequência, o motivo de menor frequência foi recusa a cirurgia $9(1,6 \%)$. As 566 cirurgias suspensas foram analisadas conforme seus respectivos motivos e os resultados foram organizados e apresentada anteriormente na Tabela 2.

\section{DISCUSSÃO}

O hospital pesquisado apresentou taxa de suspensão de $22,3 \%$ no ano de 2019 , do qual representa um índice inferior se comparado com um estudo de 2015 em um hospital público, também do distrito federal, onde teve $33,8 \%$ de taxa de suspensão ${ }^{7}$. Entretanto, ao comparar com demais pesquisas nacionais, a taxa de suspensão do hospital pesquisado mostra-se ainda dentro da média encontrada em demais estudos, que variam entre $5,2 \%^{8}$ a $27,4 \% 1$. O resultado da taxa de suspensão mostra-se bem próxima de um estudo publicado em 2019, referente às suspensões cirúrgicas de dois grandes hospitais do nordeste brasileiro, sendo um público-privado e outro público, onde o hospital público-privado apresentou taxa de suspensão de $23,13 \%$, e divergente do hospital público $42,27 \%{ }^{4}$.

Como observado na Figura 1, a respeito dos meses, o mês de novembro teve a maior incidência com 33,9\% de suspensão, neste mês muitas suspensões foram justificadas por problemas de manutenção no setor, como por exemplo: equipamentos de raio-x, escopia, e ar condicionado em manutenção, e falta de energia elétrica. Este resultado diverge do estudo realizado em um hospital do Distrito Federal que apresentou os meses de junho, julho e 
agosto com $38,5 \%$.

No que diz respeito a variável sexo do paciente, as taxas de cancelamento foram próximas para ambos os sexos. Apesar de haver diferenças nos meses isoladamente, na análise final elas se compensam e os valores tornam-se próximos, totalizou-se 297 (52,5\%) do sexo feminino, e $269(47,5 \%)$ do sexo masculino. Esse resultado é compatível com demais estudos que apresentaram taxas de $27,0 \%$ e $27,6 \%$, respectivamente, ou seja, taxas bem próximas ${ }^{1}$.

Em relação à faixa etária, a categoria dos adultos (19 a 59 anos) obteve a maioria, totalizando $362(64,0 \%)$ dos procedimentos suspensos, seguido dos idosos ( $\geq 60$ anos) 184 $(32,5 \%)$. As menores prevalência ficou por conta das crianças $(\leq 12$ anos) $14(2,5 \%)$, e adolescentes (13 a 18 anos) $6(1,1 \%)$. Este resultado pode ser justificado pelo hospital de estudo ser mais referência para a população adulto e/ou idosa, do que a infantil e/ou adolescente.

Tabela 2: Distribuição das causas de suspensões cirúrgicas, segundo as condições pessoais, clínicas e institucionais, em um hospital público do Distrito Federal, Brasília, 2020.

\begin{tabular}{|c|c|c|c|}
\hline \multicolumn{2}{|c|}{ Causas de suspensões de cirurgias } & n & $\%$ \\
\hline \multirow{7}{*}{$\begin{array}{l}\text { Causas } \\
\text { relacionadas } \\
\text { paciente }\end{array}$} & $\begin{array}{l}\text { Condição clínica } \\
\text { desfavorável }\end{array}$ & 82 & 14,5 \\
\hline & Não comparecimento & 50 & 8,8 \\
\hline & Jejum inadequado & 19 & 3,4 \\
\hline & Recusa a cirurgia & 9 & 1,6 \\
\hline & Falta de exames & 16 & 2,8 \\
\hline & Outros & 11 & 1,9 \\
\hline & Subtotal & 187 & 33,0 \\
\hline & Erro na programação & 28 & 4,9 \\
\hline
\end{tabular}




\begin{tabular}{|c|c|c|c|}
\hline \multirow{13}{*}{$\begin{array}{l}\text { Causas relacionadas à } \\
\text { unidade de saúde }\end{array}$} & Falta de leito na enfermaria & 18 & 3,2 \\
\hline & Falta de UTI & 27 & 4,8 \\
\hline & $\begin{array}{l}\text { Substituição de } \\
\text { paciente }\end{array}$ & 46 & 8,1 \\
\hline & Falta de anestesista & 27 & 4,8 \\
\hline & Avanço de horário & 128 & 22,6 \\
\hline & Falta de cirurgião & 37 & 6,5 \\
\hline & Mudança de conduta médica & 8 & 1,4 \\
\hline & Falta de material CME & 11 & 1,9 \\
\hline & $\begin{array}{l}\text { Falta de material } \\
\text { farmácia }\end{array}$ & 2 & 0,4 \\
\hline & Falta de material não especificado & 16 & 2,8 \\
\hline & Outros & 31 & 5,5 \\
\hline & Subtotal & 379 & 67,0 \\
\hline & TOTAL & 566 & 100 \\
\hline
\end{tabular}

Quanto às especialidades cirúrgicas, foram analisadas 09 especialidades, dentre elas, a ortopedia apresentou o maior índice de suspensão cirúrgica, tanto quando se considera o número de cirurgias agendadas (671 agendados e 243 suspensos, taxa de 36,2\%), como, quando considerado o total de suspensões no ano, a mesma apresentou 243 (42,9\%) procedimentos cancelados das $566(100 \%)$ suspensões, seguida pela cirurgia geral com 117 $(20,7 \%)$ procedimentos suspensos. Também quando considerado somente o número de cirurgias agendadas da clínica de forma isolada, a cirurgia geral também ocupou o $2^{\circ}$ lugar, com 527 procedimentos agendados e $117(22,2 \%)$ suspensos, nota-se que a taxa foi até 
superior comparada à taxa total de $566(100 \%)$ suspensões.

As demais especialidades mostraram taxas diferentes quando calculadas segundo o número total de suspensões, e quando calculadas de acordo com o número de cirurgias agendadas para suas clínicas de forma isolada. Este fato se justifica por certas clínicas terem mais dias de centro cirúrgico do que outras clínicas, seleção essa que varia conforme colegiado realizado na instituição hospitalar. O resultado comparativo pode ser observado na Figura 2.

Figura 2: Comparativo das taxas de suspensões relacionados às especialidades, quando considerado o valor de procedimento agendado de cada clínica de forma isolada e quando calculado de acordo com o total de suspensões de forma geral, em um hospital regional do Distrito Federal no ano de 2019.

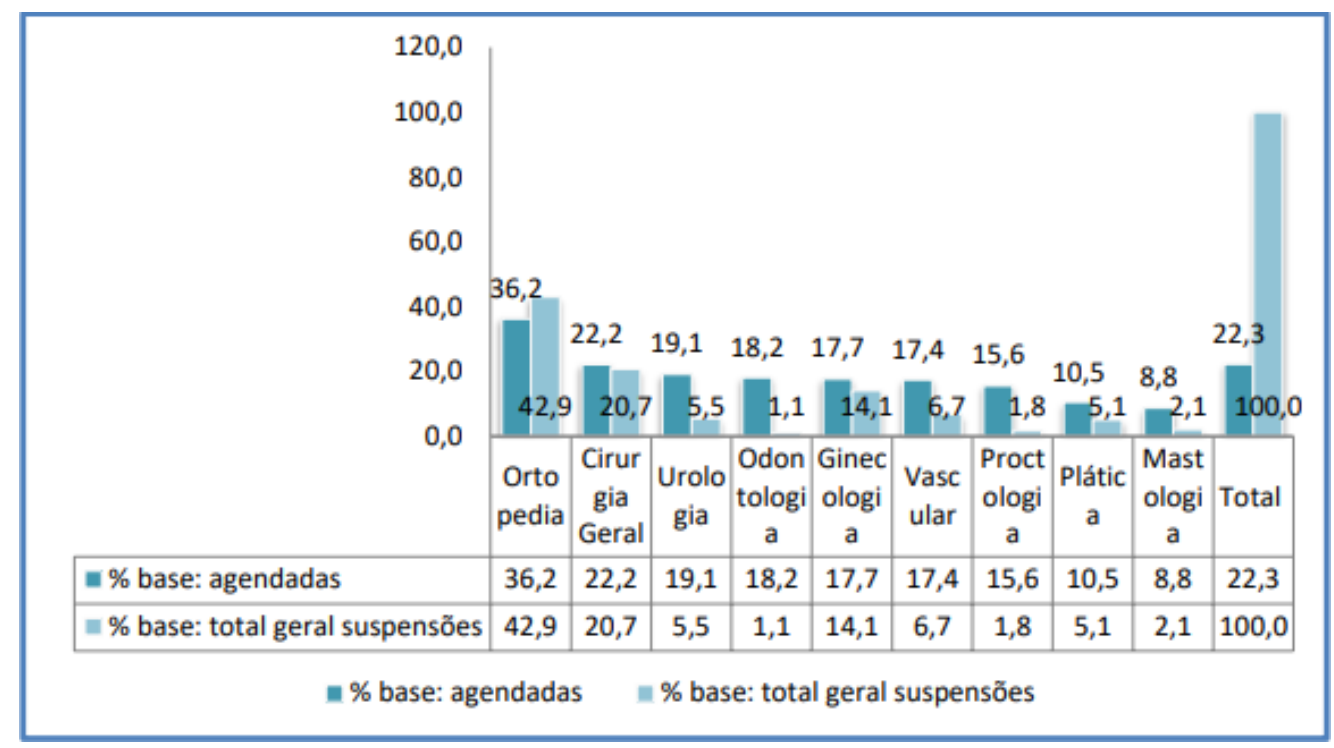

Entretanto, este resultado é superior a demais estudos realizados com o mesmo objetivo desta pesquisa, como por exemplo, um estudo realizado no sul de minas, que avaliou um período de três meses, apontou a ortopedia com incidência maior nas suspensões, apresentando $133(28,5 \%)$ de um total de 466 (100\%) cirurgias suspensas ${ }^{1}$, diferente de outro estudo realizado no período de fevereiro de 2015 a fevereiro de 2016, onde obteve 9.648 cirurgias eletivas e destas $2.956(30,6 \%)$ suspensas, ressaltou a incidência da cirurgia geral em 
primeiro lugar nas suspensões, com taxas de $29,8 \%$, seguida da ortopedia com $17,6 \%$. Portanto, vale ressaltar que as especialidades da ortopedia e cirurgia geral estão em incidência na maioria dos estudos ${ }^{4,11}$.

Em relação aos fatores clínicos e estruturais, ou seja, relacionados ao paciente e/ou a unidade, apresentaram taxas de 187 (33,0\%) e 379 (67,0\%), respectivamente. Nota-se que os fatores estruturais são superiores aos clínicos, que estão diretamente ligados ao paciente. Sendo assim, como medida para a minimização do problema a literatura cita que é consenso entre os autores que a existência de ambulatórios de anestesia e visitas pré-operatórias reduzem o número de suspensões cirúrgicas, por permitirem a previsão de possíveis complicações clínicas ${ }^{5}$.

Entretanto, como observado anteriormente na Tabela 2, podemos da ênfase as causas relacionadas ao paciente de maior prevalência que foram: a condição clínica desfavorável 82 (14,5\%), seguida do não comparecimento do paciente $50(8,8 \%)$, este resultado é semelhante a estudos anteriores, que apresentou taxas entre $19,6 \%$ a $20,8 \%$ e $26,9 \%$ a $22,7 \%$, respectivamente ${ }^{4,5}$

Já os fatores estruturais tomaram destaque neste estudo, das 566 suspensões, 379 (67,0\%) foram relacionados à unidade de saúde. Assim também como observado na Tabela 2, os motivos de maior destaque foram: Avanço de horário 128 (22,6\%), substituição de pacientes $46(8,1 \%)$, e falta de cirurgião 37 (6,5\%). Com relação ao avanço de horário e substituição de paciente a somatória 30,7\% é compatível com estudo realizado em 2015, em um hospital do Distrito Federal também, que cita a ocorrência dos mesmos em primeiro lugar, classificando-os como causas injustificadas e alcançando taxa de $30,1 \%^{7}$. Quanto à falta de cirurgião o resultado deste estudo é compatível com um estudo de 2014 e 2015 que comparou dois hospitais, sendo um privado-público e o outro público, que apresentou taxas de 5,3\% e $11,7 \%$ em 2014 , e $7,2 \%$ e $8,7 \%$ em 2015 , respectivamente ${ }^{4}$. 
Em virtude dos resultados encontrados e com o intuito de pontuar as causas mais frequentes com as respectivas especialidades, a fim de identificar respostas e propor melhorias, elaboramos a Tabela 3, onde podemos observar às causas de suspensões de acordo com o paciente e de acordo com a unidade de saúde, e correlaciona-los as especialidades. No que diz respeito aos fatores relacionados ao paciente a causa de maior incidência, que foi condições clínicas desfavoráveis, está mais relacionada com a especialidade da ortopedia 48 (25,7\%), seguido pela cirurgia geral 39 (20,9\%). Tal motivo esse justificado muitas vezes e correlacionado com exames alterados ou vencidos, e/ou uso de medicações contraindicadas no pré-operatório.

Este resultado conforme podemos encontrar na literatura, ocorre por falhas nas visitas pré-operatórias, construção inadequada do mapa cirúrgico, como tempo de antecedência de sua construção, falta de programação por parte do cirurgião, e longas filas de espera ocasionando o vencimento dos exames pré-operatórios. Todos esses fatores são de possível resolução e impactariam diretamente na redução das taxas de suspensão cirúrgica ${ }^{5}$.

Tabela 3: Correlações das causas de suspensões com as respectivas especialidades.

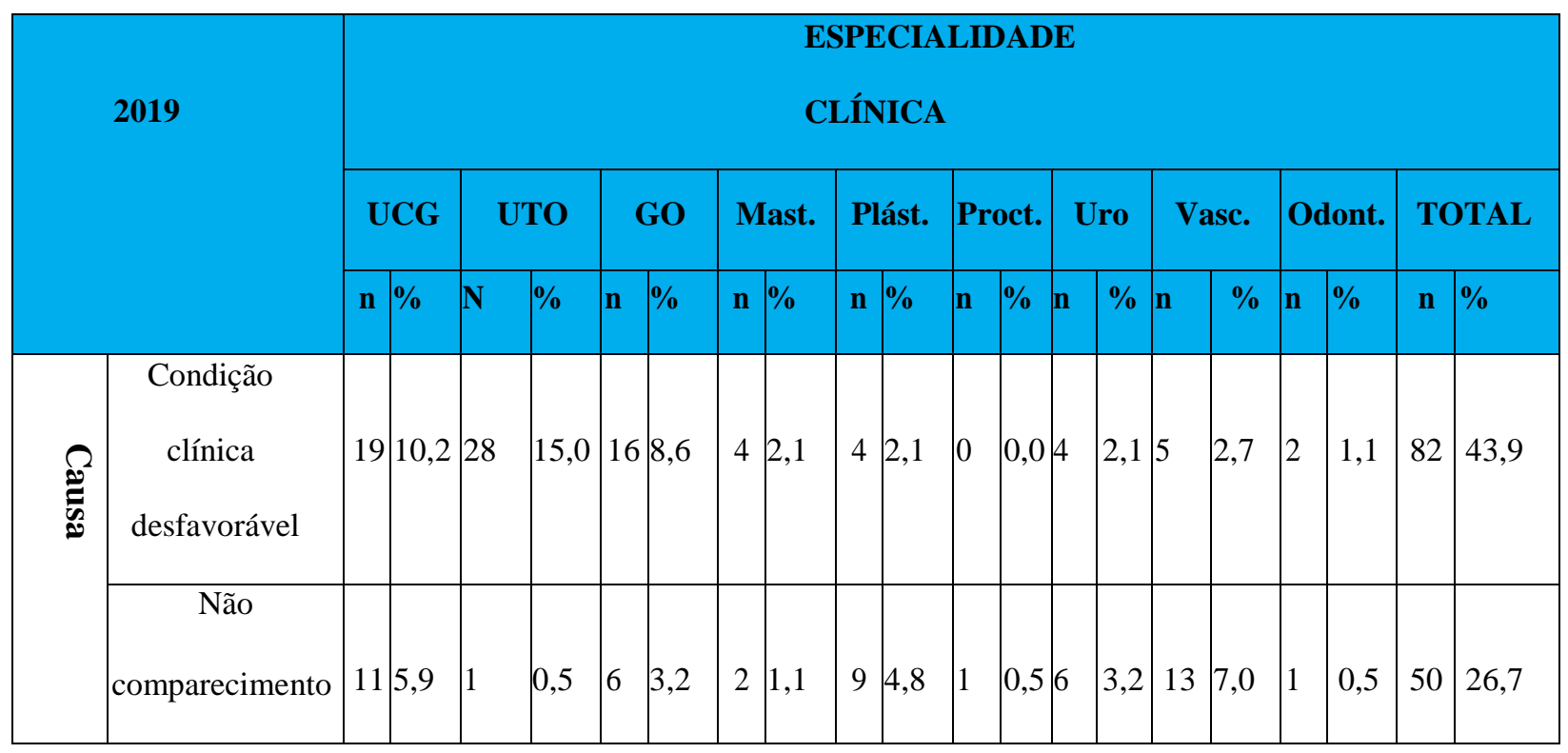




\begin{tabular}{|c|l|l|l|l|l|l|l|l|l|l|l|l|l|l|l|l|l|l|l|l|}
\hline $\begin{array}{c}\text { Jejum } \\
\text { inadequado }\end{array}$ & 2 & 1,1 & 10 & 5,3 & 1 & 0,5 & 0 & 0,0 & 4 & 2,1 & 0 & 0,0 & 0 & 0,0 & 2 & 1,1 & 0 & 0,0 & 19 & 10,2 \\
\hline Recusa a & 0 & 0,0 & 2 & 1,1 & 1 & 0,5 & 1 & 0,5 & 2 & 1,1 & 1 & 0,5 & 2 & 1,1 & 0 & 0,0 & 0 & 0,0 & 9 & 4,8 \\
cirurgia & & & & & & & & & & & & & & & & & & & & \\
\hline Falta de & 5 & 2,7 & 3 & 1,6 & 1 & 0,5 & 0 & 0,0 & 1 & 0,5 & 1 & 0,5 & 2 & 1,1 & 2 & 1,1 & 1 & 0,5 & 16 & 8,6 \\
\hline exames & & & & & & & & & & & & & & & & & & & & \\
\hline Outros & 2 & 1,1 & 4 & 2,1 & 3 & 1,6 & 0 & 0,0 & 1 & 0,5 & 1 & 0,5 & 0 & 0,0 & 0 & 0,0 & 0 & 0,0 & 11 & 5,9 \\
\hline
\end{tabular}

\section{\begin{tabular}{l|l|l|l|l|l|l|l|l|l|l|l|l|l|l|l|l|l|l|l|l|} 
SUBTOTAL & 39 & 20,9 & 48 & 25,7 & 28 & 15,0 & 7 & 3,7 & 21 & 11,2 & 4 & 2,1 & 14 & 7,5 & 22 & 11,8 & 4 & 2,1 & 187 & 100,0
\end{tabular}}

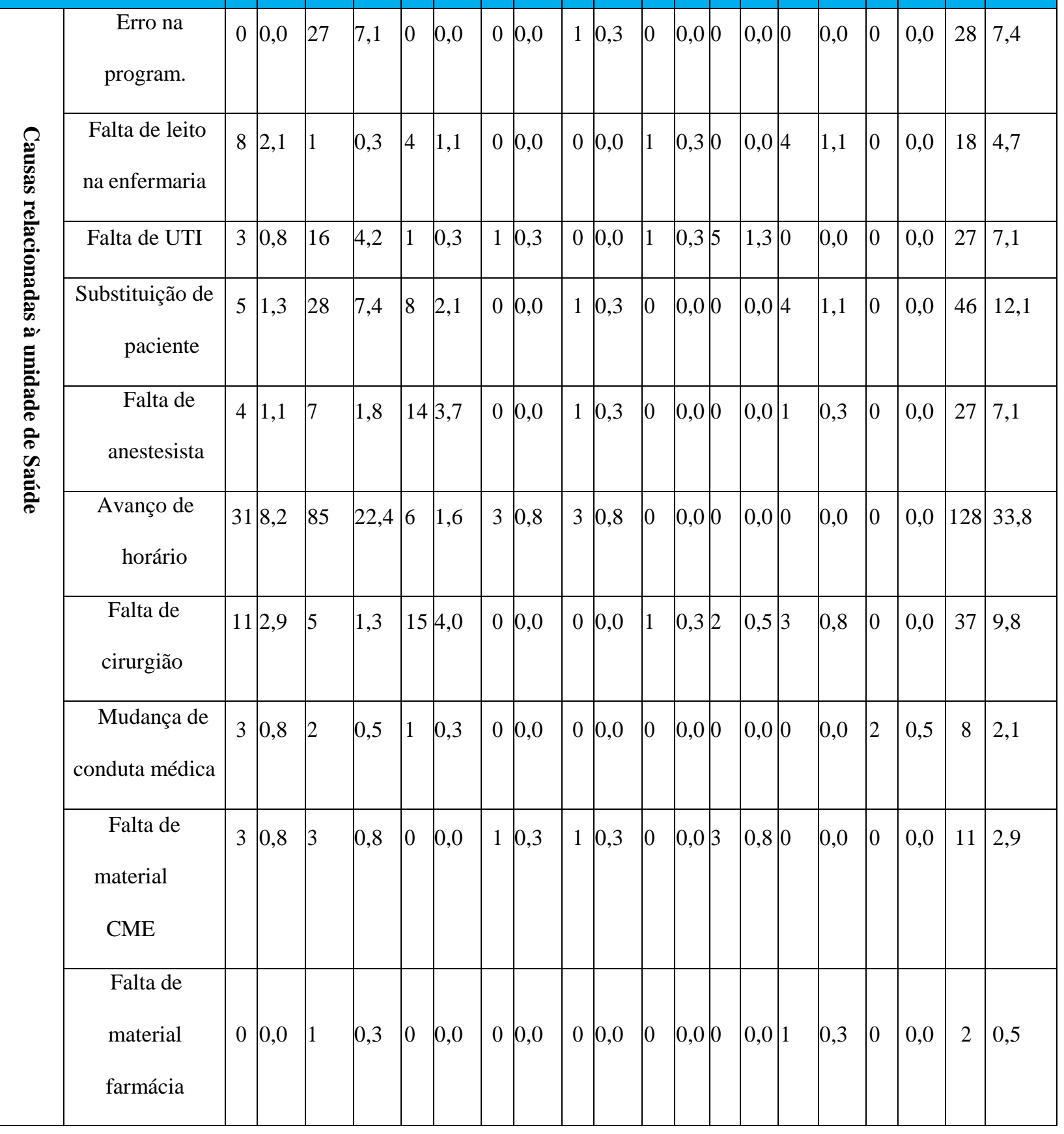




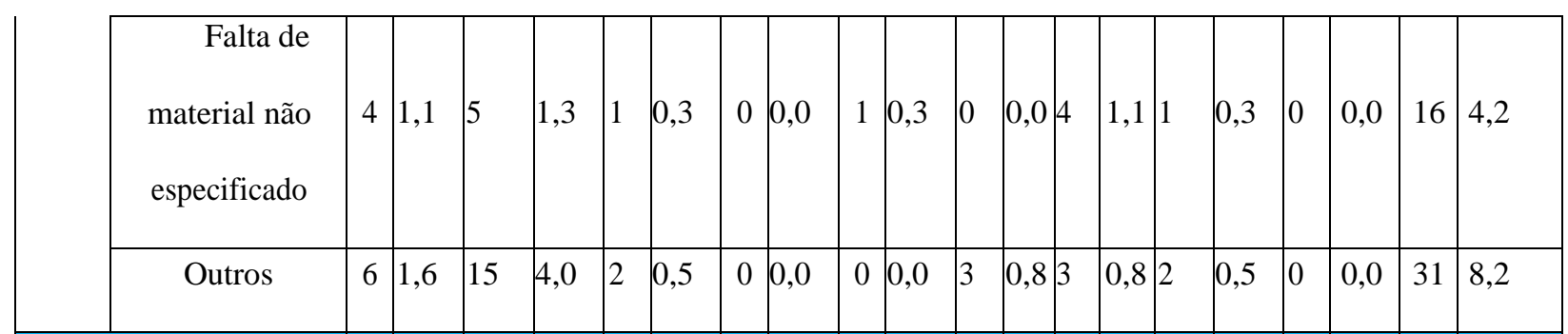

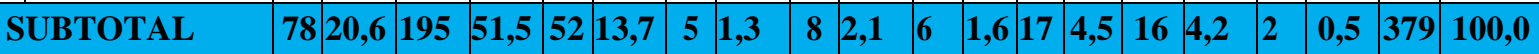

Vale ressaltar, que na maioria dos registros não especifica o que levou ao paciente estar em condições clinicas desfavoráveis, dados esses de extrema importância para que seja possível identificar e intervir. Esse achado mostra a necessidade de conscientização dos profissionais, pois apenas a partir do conhecimento da causa é que se consegue propor medidas para correção da fragilidade.

Em relação aos fatores estruturais, ou seja, relacionados à unidade de saúde, o motivo de maior destaque foi avanço de horário, das 128 (33,8\%) das ocorrências, 85 (22,4\%) delas estão correlacionadas à especialidade da ortopedia, seguido por $31(8,2 \%)$ da cirurgia geral. O segundo maior motivo foi à substituição de pacientes das $46(12,1 \%), 28(7,4 \%)$ está correlacionado a especialidade da ortopedia, seguido pela ginecologia com 8 (2,1\%). Este resultado da ortopedia muitas vezes foi justificado por falta de material, ou até mesmo equipamentos como escopias e RX que no momento da cirurgia estavam em manutenção, essas justificativas quando mencionadas, foram incluídas na categoria Outros que corresponde respectivamente a 15 (4,0\%) das substituições. Resultado semelhante ao estudo realizado em 2014 e $2015^{4}$, e ao estudo realizado no Distrito Federal em $2015^{7}$.

Em relação ao quantitativo geral a falta de cirurgião citada anteriormente, também teve um quantitativo relevante, das $37(9,8 \%)$ suspensões, $15(4,0 \%)$ corresponde à especialidade da ginecologia, justificadas muitas vezes por atestados médicos, ou falta de anestesista em certo período do ano. Os demais motivos podem ser observados na Tabela 3. Estes resultados de forma geral são bastante condizentes com demais estudos já citados anteriormente, observa-se valores para 
mais ou para menos, mas que estão correlacionados com as mesmas categorias ${ }^{4,7}$.

Portanto, para a minimização das suspensões a literatura traz a necessidade quanto à confirmação do agendamento em um período próximo ao da cirurgia, reuniões periódicas para discussão e planejamento das cirurgias futuras, visita pré- ambulatorial, aprimoramento da comunicação entre instituição e usuários, realização de visita pré-anestésica ambulatorial e monitoramento dos indicadores a fim de controlar e investigar os motivos de suspensão. Desta forma, incluem-se os indicadores de suspensões cirúrgicos como avaliação para melhoria do serviço prestado nos centros cirúrgicos ${ }^{1}$.

As limitações da pesquisa foram à falta de algumas informações que justificasse de forma completa a suspensão, como por exemplo, o que caracterizou a falta de condição clínica, qual material de fato faltou, e entre outros. Havia alguns registros sem as devidas informações, o que ocasionou uma busca ativa no Sistema TrackCare a fim de obter a informação desejada. Observa- se que é pertinente a realização de um trabalho acerca da operacionalização do CC, a fim de possibilitar à instituição uma análise mais precisa a respeito da produtividade, como por exemplo, o mapa cirúrgico no sistema TrackCare, assim forçaria o registro completo das cirurgias, e evitaria de passar despercebido o registro de certas cirurgias.

\section{CONCLUSÃO}

A pesquisa permitiu realizar uma análise situacional de um hospital público do Distrito Federal, o mesmo apresentou uma taxa de suspensão cirúrgica semelhante a encontradas em alguns estudos nacionais, e inferior a um estudo na mesma região no ano de 2015.

Evidenciou a maioria das suspensões no sexo feminino, quanto à faixa etária os adultos (19 a 59 anos) tomaram destaque. As maiores frequências de suspensão cirúrgica ocorreram na ortopedia e cirurgia geral, com dados até superior a outros estudos, já com a menor taxa destacou-se a mastologia. Os fatores determinantes para suspensões de cirurgias tiveram maior prevalência 
quando relacionados à unidade de saúde, tendo como maiores frequências os seguintes motivos: avanço de horário, substituições de pacientes, e falta de cirurgião. Os motivos de falta de material da farmácia e mudança de conduta médica tiveram as menores taxas. Quanto aos fatores relacionados ao paciente, a falta de condição clínica e o não comparecimento obtiveram maiores frequências, em contrapartida a recusa a cirurgia obteve a menor taxa.

Com estes resultados, o intuito é conscientizar toda a equipe assistencial e administrativa ressaltando a importância do registro de informações fidedignas e completas relacionadas às suspensões cirúrgicas, para que assim tenha um monitoramento dos indicadores de suspensão cirúrgica. Vale ressaltar que o mesmo deve ser contínuo, ser apresentado aos gestores do setor para que assim todos tenham uma visão geral e desta forma subsidiar o planejamento de estratégias de intervenção com vistas à redução da taxa de suspensão, aumento da qualidade do serviço prestado e consequente minimização dos transtornos causados para os clientes, familiares e instituição.

\section{REFERÊNCIAS}

1. Botazini NO, Toledo LD, Souza DMST. Cirurgias eletivas: cancelamentos e causas. Rev SOBECC [periódico na Internet]. 2015 [acessado 2020 Set 01];20(4):210-9. doi: $\underline{10.5327 / Z 1414-4425201500040005 .}$

2. Sociedade Brasileira de Enfermeiros de Centro Cirúrgico, Recuperação Anestésica e Centro de Material e Esterilização (SOBECC). Diretrizes de práticas em enfermagem cirúrgica e processamento de produtos para a saúde. 7. ed. São Paulo: SOBECC,2017.

3. Garcia ACKA, Fonseca LF. A Problemática da suspensão cirúrgica: a perspectiva dos anestesiologistas. Rev enferm UFPE online [periódico na Internet]. 2013 Fev [acessado 2020 Ago 24];7(2):481-90. Disponível em: https://periodicos.ufpe.br/revistas/revistaenfermagem/article/view/10258/1087\%209 
4. Araujo JKM, Ferreira FAS, Comassetto I, Bernado THL. Avaliação dos fatores de cancelamento de cirurgias em hospitais do Nordeste Brasileiro. Rev SOBECC [periódico na Internet]. 2019 Out-Dez [acessado 2020 Ago 24];24(4):175-184. Disponível em: http://www.dx.doi.10.5327/Z1414-4425201900040002.

5. Carvalho TA, Sobral CB, Marinho PML, Rodriguez EOOL, Campos MPA. Suspensões de Cirurgias em um Hospital Universitário. Rev SOBECC [periódico na Internet]. 2016 Out- Dez [acessado 2020 Ago 24];21(4):186-191. Disponível em: http://www.dx.doi.10.5327/Z1414$\underline{4425201600040002 .}$

6. Moraes PGS, Pachêco NMD, Souza RG, Silva PCV. Fatores clínicos e organizacionais relacionados à suspensão de procedimentos cirúrgicos. Rev de Enf UFPE Online [periódico na internet]. Jul 2017 [acessado 2020 Ago 25];11(7):2645-53. Disponível em: https://periodicos.ufpe.br/revistas/revistaenfermagem/article/view/23436/19132.

7. Gomes JRAA, Franco RVB, Morais DSVD, Barbosa BC. Fatores determinantes para suspensões de cirurgias eletivas em um hospital do Distrito Federal. Rev SOBECC [periódico na Internet]. 2018 Out-Dez [acessado 2020 Ago 25];23(4):184-8. Disponível em: https://www.revista.sobecc.org.br/sobecc/article/view/419/pdf.

8. Moreira LR, Xavier APZ, Moreira FN, Souza LCM, Araujo OC, Santos TMB, et al. Avaliação dos motivos de cancelamento de cirurgias eletivas. Enfermagem Revista [periódico na Internet]. Out 2016 [acessado 2020 Ago 26];19(2):212-225. Disponível em: http://periodicos.pucminas.br/index.php/enfermagemrevista/article/view/13156

9. Prodanov AA, Freitas EC. Metodologia do trabalho cientifico: métodos e técnicas da pesquisa e do trabalho acadêmico. [periódico na Internet]. ed. $2^{\mathrm{a}}$. Novo Hamburgo: Feevale; 2013 [acessado 2020 Ago 30]. 277 p. ISBN: 9788577171583. Disponível em: http://www.feevale.br/Comum/midias/8807f05a-14d0-4d5b-b1ad1538f3aef538/Ebook\%20Metodologia\%20do\%20Trabalho\%20Cientifico.pdf 
10. Brasil. Ministério da Saúde. Conselho Nacional de Saúde. Resolução 466, de 12 de dezembro de 2012. Aprova as diretrizes e normas regulamentadoras de pesquisas envolvendo seres humanos. Diário Oficial da União [Internet]. 2013 Jun 13 [acessado 2021 Jun 1]. (Seção 1):59. Disponível em: http://conselho.saude.gov.br/resolucoes/2012/Reso466.pdf

11. Macedo JM, Kano JA, Braga EM, Garcia MA, Caldeira SM. Cancelamento de cirurgias em um hospital universitário: causas e tempo de espera paranovo procedimento. Rev SOBECC [periódico na Internet] 2013 Jan-Mar [acessado 2020 Set 01];18(1):26-34. Disponível em: $\quad$ http://www.sobecc.org.br/arquivos/artigos/2012/pdf/ArtigosCientificos/Ano18_n1_jan_mar2013_cancelamento-de-cirurgias-em-umhospitaluniversitario.pdf. 